\title{
Assessment of the Pacific red snapper (Lutjanus peru) fishery in the southwestern Gulf of California
}

\section{Evaluación de la pesquería del huachinango (Lutjanus peru) en el suroeste del Golfo de California}

\author{
J. Gabriel Díaz-Uribe ${ }^{\mathrm{a}}$ \\ Ernesto A. Chávez ${ }^{2}$ \\ Juan F. Elorduy-Garay ${ }^{2}$ \\ ${ }^{1}$ Centro de Estudios Sustentables \\ Universidad Autónoma de Baja California Sur \\ Apartado postal 19-B \\ La Paz, CP 23080, Baja California.Sur, México \\ E-mail: diazjuan@prodigy.net.mx \\ ${ }^{2}$ Departamento de Pesquerías y Biología Marina \\ Centro Interdisciplinario de Ciencias Marinas \\ Instituto Politécnico Nacional \\ Apartado postal 592 \\ La Paz, CP 23000, Baja California.Sur, México
}

Recibido en julio de 2003; aceptado en mayo de 2004

\begin{abstract}
The Pacific red snapper (Lutjanus peru) fishery in the southwestern Gulf of California was analyzed with an age-structured simulation model. In addition to natural mortality $(M)$ and fishing mortality $(F)$, the model incorporates juvenile by-catch mortality $(f)$ caused by the shrimp fishery in the zone. Four simulated scenarios were tested in which different levels of mortality were combined. Contrary to what is established in the National Fishing Charter, simulation outputs showed that the Pacific red snapper fishery cannot be developed if both $F$ and $f$ are kept unregulated as presently occurs. According to the model, the fishery could only be developed with an effective regulation of these mortality sources, which implies control measures for both the artisanal fleet and the shrimp fleet.
\end{abstract}

Key words: Lutjanidae, Pacific red snapper, stock assessment, Gulf of California, small-scale fisheries.

\section{Resumen}

Se analizó la pesquería del huachinango (Lutjanus peru) en la región suroeste del Golfo de California con un modelo de simulación estructurado por edades. Además de la mortalidad natural $(M)$ y la mortalidad por pesca $(F)$, el modelo incorpora la mortalidad incidental de juveniles $(f)$ que se da por la pesca de camarón en la zona. Se probaron cuatro escenarios de simulación en donde se combinaron diferentes niveles de mortalidad. Contrario a lo que señala la Carta Nacional Pesquera, los resultados del modelo mostraron que la pesquería no tiene posibilidades de desarrollarse si $F$ y $f$ continúan sin ser reguladas como hasta ahora. De acuerdo al modelo, la pesquería podría desarrollarse mediante una regulación efectiva de ambas fuentes de mortalidad, lo que implica medidas de control tanto para la flota artesanal, como para la flota camaronera.

Palabras clave: Lutjanidae, huachinango, evaluación pesquera, Golfo de California, pesquerías artesanales.

${ }^{a}$ Current Address: Instituto Nacional de la Pesca. Centro Regional de Investigación Pesquera - La Paz, Carretera a Pichilingue Km. 1, La Paz 23020, BCS, Mexico

\section{Introduction}

The Pacific red snapper (Lutjanus peru) (fig. 1) is one of the most important fish resources landed by artisanal fleets along the Mexican Pacific coast, both by catch volume and by commercial value (INP, 2001). In some northwestern regions of Mexico, such as the eastern coast of Baja California Sur, the multi-species fishing cycle mainly depends on this species

\section{Introducción}

De los recursos de escama que desembarcan las flotas artesanales en el Pacífico mexicano, el huachinango (Lutjanus peru) (fig. 1) es una de las especies más importantes tanto por su volumen de capturas como por su valor comercial (INP, 2001). En algunas regiones del noroeste mexicano, como la costa este de Baja California Sur, el ciclo de pesca depende en 
because, according to the landing records of 1998-2000, Pacific red snapper can contribute up to $25 \%$ of the income by first-sales at the beach.

Despite its importance, assessment studies for the Pacific red snapper are so scarce that the status of the fishery is not clear. In Nayarit and Colima, the fishery appears to be below its maximum yield (Cruz-Romero et al., 1996; Del MonteLuna et al., 2001), while there is evidence of overexploitation in Guerrero (Santamaría and Chávez, 1999). In some official documents, the Pacific red snapper fishery is considered to have potential for development along the Pacific coast (SEMARNAP, 2000a; INP, 2001); however, the National Fishing Charter (Carta Nacional Pesquera) states that the fishery only has potential for development in the state of Baja California Sur (BCS), because in the rest of the country it is captured at its maximum sustainable yield (Diario Oficial de la Federación, August 28, 2000).

Without previous assessment studies for this fishery in BCS, the potential for development can be called into question. For instance, in the bays of La Paz and La Ventana (fig. 2), where the Pacific red snapper fishery is most productive in BCS, nearly $50 \%$ of the commercial landings are composed of fish under $40 \mathrm{~cm}$, and $94 \%$ of them are sexually immature (Rocha-Olivares, 1991). Moreover, juveniles have been recorded in the shrimp fishery by-catch (Pérez-Mellado and Findley, 1985); therefore, in this area, where both fisheries operate (Ross-Terrazas, 1988), the incidental mortality of juveniles is an important factor affecting the dynamics of the population. The purpose of this work is to evaluate the state of the Pacific red snapper fishery in the bays of $\mathrm{La} \mathrm{Paz}$ and $\mathrm{La}$ Ventana through a simulation model that integrates the effect of juvenile mortality.

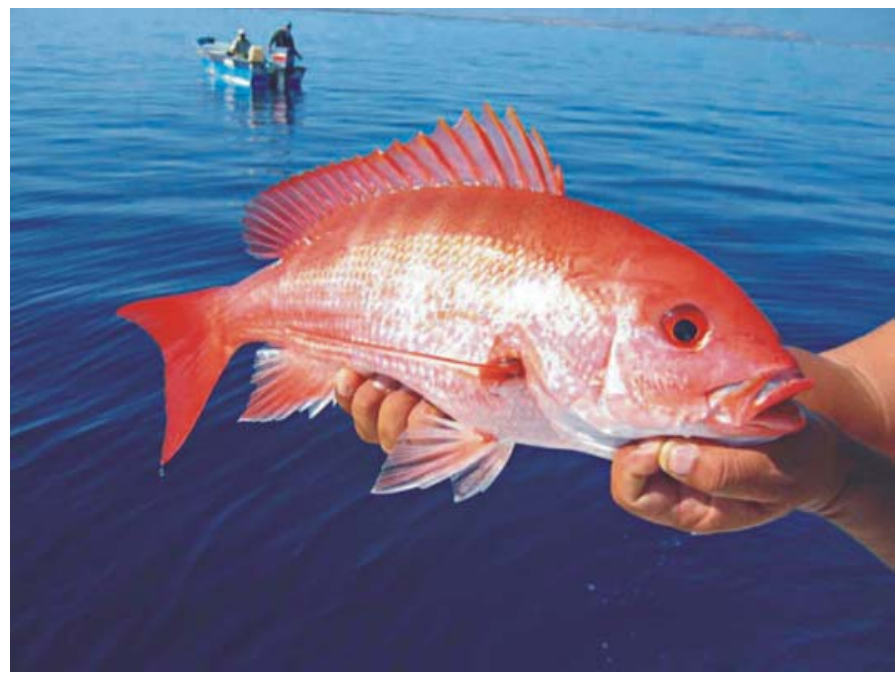

Figure 1. Pacific red snapper (Lutjanus peru) caught off Baja California Sur (Mexico). Photo by Stan Moberly, taken from http://www.mexfish.com.

Figura 1. Huachinango (Lutjanus peru) capturado en aguas de Baja California Sur (México). Fotografía de Stan Moberly, tomada de http:// www.mexfish.com. gran medida de esta especie ya que, según los avisos de arribo de 1998-2000, de las múltiples especies que se capturan el huachinango puede aportar hasta $25 \%$ de los ingresos por ventas en playa.

A pesar de su importancia, son pocos los estudios de evaluación pesquera que se han hecho para el huachinango y en consecuencia el estatus de la pesquería es confuso. En Nayarit y Colima la pesquería parece estar por debajo de su rendimiento máximo (Cruz-Romero et al., 1996; Del Monte-Luna et al., 2001), en cambio, en Guerrero existen evidencias de que la pesquería está sobrexplotada (Santamaría y Chávez, 1999). Sobre esta base, en algunos documentos oficiales se considera que la pesquería del huachinango en el Pacífico tiene potencial de desarrollo (SEMARNAP, 2000a; INP, 2001). Sin embargo, en la Carta Nacional Pesquera se establece que sólo en Baja California Sur (BCS) la pesquería tiene potencial de desarrollo, mientras que en el resto del país está aprovechada al máximo sustentable (Diario Oficial de la Federación, 28 de agosto de 2000).

El problema es que en BCS no existen antecedentes de evaluación de la pesquería y, por el contrario, hay evidencias indirectas que cuestionan su potencial de desarrollo. Por ejemplo, en las bahías de La Paz y La Ventana (fig. 2), una de las regiones más productivas en huachinango en el estado, aproximadamente $50 \%$ de las capturas comerciales se

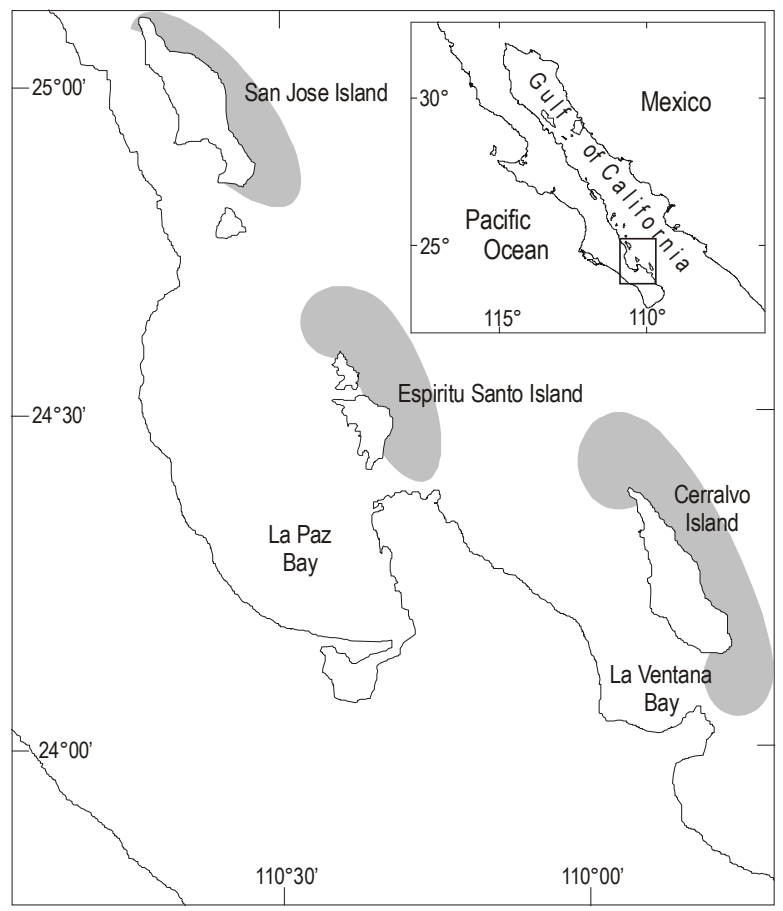

Figure 2. Map of the study area. Shaded areas indicate the zones where Pacific red snapper are commercially caught and biological samples were collected.

Figura 2. Mapa del área de estudio. El área sombreada señala las zonas donde se captura comercialmente el huachinango y donde se obtuvieron las muestras biológicas. 


\section{Methods}

\section{Basic parameters}

From March 1989 to February 1991, 1230 specimens were collected from commercial landings at the bays of La Paz and La Ventana (fig. 2). Length, weight and sex were determined for each specimen. The age at first maturity $\left(t_{m}\right)$ and the age at first catch $\left(t_{c}\right)$ were estimated with the growth parameters and the length-age key estimated by Rocha-Olivares (1998). Total mortality $(Z)$ was estimated by fitting the exponential decay model to the descending limb of the age-structure. Natural mortality $(M)$ was calculated by the methods of Pauly (1980), Ralston (1987) and Jensen (1997), and for further analysis, the median of these estimates was used. When it was needed, an environmental temperature of $17^{\circ} \mathrm{C}$ (Lavín et al., 1997) was used. Fishing mortality $(F)$ was calculated by subtracting $M$ from $Z$. Catch records for 1978-1982 were obtained from official annual fishing statistics for the study area. Catch records for 1983-2000 were obtained from the landing records fishermen deliver at fisheries offices in their locality.

\section{Age-structure reconstruction}

The catch number per age group in a given year was estimated using the age-length key, the growth parameters and the length-weight relationship. Thereafter, population abundance at each age was estimated using the catch equation:

$$
C_{a}=N_{a} \frac{F}{(F+M)}\left(1-\mathrm{e}^{-(F+M)}\right)
$$

or

$$
N_{a}=\frac{(F+M)}{F} \cdot \frac{C_{a}}{\left(1-\mathrm{e}^{-(F+M)}\right)}
$$

where $C_{a}$ is the number of fish caught at age $a, N_{a}$ is the number of fish at age $a, F$ is the fishing mortality rate, and $M$ is the natural mortality rate. This routine was done for the historical catch series, assuming a stable age structure in the population. Fine-tuning was done by fitting the exponential decay model to each cohort present in the time series. The age structure with the highest estimated abundance is hereafter referred to as the initial age structure (IAS) and it was used as an input for the simulation model described below.

\section{Recruitment parameters}

With the reconstructed age structure in the historical series, the Beverton and Holt recruitment model was fitted as follows:

$$
R_{y+1}=\frac{a^{\prime} S_{y}}{b^{\prime}+S_{y}}
$$

where $R_{y+1}$ is the number of recruits at age 1 in year $y+1$, $S_{y}$ is the number of adults in year $y, a^{\prime}$ is the maximum number componen de organismos $<40 \mathrm{~cm}$ y de ellos, 94\% son sexualmente inmaduros (Rocha-Olivares, 1991). Además, los juveniles de esta especie forman parte de la fauna de acompañamiento en la pesquería del camarón (Pérez-Mellado y Findley, 1985) y, por lo tanto, en esta zona donde concurren ambas pesquerías (Ross-Terrazas, 1988) la mortalidad incidental de juveniles es un factor que afecta de manera importante la dinámica de la población. El propósito de este trabajo es evaluar el estado de la pesquería artesanal del huachinango en las bahías de La Paz y La Ventana a través de un modelo de simulación que integra el efecto de la mortalidad de juveniles.

\section{Métodos}

\section{Parámetros básicos}

De marzo de 1989 a febrero de 1991 se recolectaron 1230 individuos de los desembarcos comerciales de las bahías de $\mathrm{La}$ Paz y La Ventana (fig. 2). Cada organismo se midió, se pesó y se le determinó el sexo. Con los parámetros de crecimiento y la clave edad-talla estimados por Rocha-Olivares (1998) se estimaron la edad de primera madurez $\left(t_{m}\right)$ y la edad de primera captura $\left(t_{c}\right)$. La mortalidad total $(Z)$ se estimó ajustando el modelo de decaimiento exponencial a la curva descendente de la estructura de edades. La mortalidad natural $(M)$ se calculó con los métodos de Pauly (1980), Ralston (1987) y Jensen (1997), y para el análisis posterior se utilizó la mediana de estas estimaciones. Cuando fue necesario se consideró una temperatura ambiental media de $17^{\circ} \mathrm{C}$ (Lavín et al., 1997). La mortalidad por pesca $(F)$ se calculó por la diferencia entre $Z$ y $M$. Los registros de captura de 1978-1982 se obtuvieron de los anuarios estadísticos de pesca, en los cuales se desglosa la captura de la zona de estudio. Las capturas de 1983-2000 se obtuvieron de los avisos de arribo que los pescadores entregan a la oficina de pesca de cada localidad.

\section{Reconstrucción de la estructura de edades}

Con la clave edad-talla, los parámetros de crecimiento y la relación longitud-peso se estimó el número de organismos capturados por grupo de edad en un año determinado. A partir de esta información se estimó la abundancia de la población a cada edad con la ecuación de captura:

$$
C_{a}=N_{a} \frac{F}{(F+M)}\left(1-\mathrm{e}^{-(F+M)}\right)
$$

o bien

$$
N_{a}=\frac{(F+M)}{F} \cdot \frac{C_{a}}{\left(1-\mathrm{e}^{-(F+M)}\right)}
$$

donde $C_{a}$ es el número de organismos capturados a la edad $a$, $N_{a}$ es el número de organismos de edad $a, F$ es la tasa de mortalidad por pesca y $M$ es la tasa de mortalidad natural. Esta 
of recruits, and $b^{\prime}$ is the number of adults needed to reach $a^{\prime} / 2$ recruits. Parameters $a^{\prime}$ and $b^{\prime}$ were simultaneously fitted by the Newton iteration method using the minimum squares criterion and the restriction $\log _{\mathrm{e}} a^{\prime} / b^{\prime}=1.9$ (Myers et al., 1999).

\section{Total costs and income}

Direct field inquiries were made during 1999 at different fishing camps to estimate the number of fishing trips, first-sale price and average cost per trip. Total income from first-sale at beach was obtained from the local landing records of that year.

\section{Simulation analysis}

The following exponential decay model was used:

$$
N_{a+1, y+1}=N_{a, y} \cdot \mathrm{e}^{-\left(S_{a} f+S_{a} F_{y}+M\right)}
$$

where $N_{a, y}$ is the number of fish at age $a$ at the beginning of year $y ; N_{a+1, y+1}$ is the number of fish in the cohort that survived to the next year; $f$ is the juvenile by-catch mortality rate; $F_{y}$ is the fishing mortality rate during year $y ; M$ is the natural mortality rate; and $s_{a}$ and $S_{a}$ are switches that allowed $f$ and $F_{y}$ to be applied to different age groups according to the following criteria: $s_{a}=1$ when $a<\mathrm{t}_{\mathrm{m}}$, otherwise $s_{a}=0 ; S_{a}=0$ when $a<t_{c}$, otherwise $S_{a}=1$. Recruits for a given year were calculated by equation 1 and its fitted parameters. To start the model, IAS was taken as an input and seed values were assigned to mortality rates $f$ and $F_{y}$, and thereafter the catch number was calculated by:

$$
C_{a, y}=N_{a, y} \frac{F_{y}}{\left(F_{y}+M\right)}\left(1-\mathrm{e}^{\left(-F_{y}-M\right)}\right)
$$

where $C_{a, y}$ is the number of fish at age $a$ caught in year $y$. Then the simulated annual yield was estimated by:

$$
Y_{S I M, y}=\sum_{a=t_{c}}^{k} C_{a, y} \cdot W_{a}
$$

where $Y_{\text {SIM a,y }}$ is the simulated yield for year $y, w_{a}$ is the average individual weight at age $a, t_{c}$ is the age at first capture, and $k$ is the maximum age in the population.

The abundance of each cohort was fitted year by year, varying $F_{y}$ in equation 2 until the simulated yield matched the recorded yield, that is, $Y_{S I M, y}=Y_{R E C, y}$. Since this condition was fulfilled with different values of $f$, the maximum juvenile mortality $\left(f_{\max }\right)$ that the simulated population could withstand to resemble the 1978-2000 historical catch series was evaluated. Thus, the simulation model was calibrated by fitting the values of $F_{y}$ with $f=0.5 f_{\max }$. operación se realizó para todos los años de las serie histórica, suponiendo que la población tiene una estructura de edades estable. Las estimaciones de abundancia se afinaron ajustando el modelo de decaimiento exponencial a cada cohorte presente en la serie histórica. La estructura de edades con la mayor abundancia estimada se usó como entrada del modelo de simulación que se describe más adelante y se denominará de aquí en adelante Estructura de Edades Inicial (EEI).

\section{Parámetros de reclutamiento}

Con la estructura de edad reconstruida en la serie histórica se ajustó el modelo de reclutamiento de Beverton y Holt en su forma

$$
R_{y+1}=\frac{a^{\prime} S_{y}}{b^{\prime}+S_{y}}
$$

donde $R_{y+1}$ es el número de reclutas de edad 1 en el año $y+1, S_{y}$ es el número de adultos en el año $y, a^{\prime}$ es el máximo número de reclutas, y $b^{\prime}$ es el número de adultos con el que se tienen $a^{\prime} / 2$ reclutas. Los parámetros $a^{\prime}$ y $b^{\prime}$ se ajustaron simultáneamente por el método de iteración de Newton utilizando como criterio de ajuste los mínimos cuadrados y la restricción $\log _{\mathrm{e}} a^{\prime} / b^{\prime}=1.9$ (Myers et al., 1999).

\section{Costos e ingresos totales}

Para estimar el número de viajes de pesca, el precio del huachinango en playa y el costo promedio por viaje, se realizaron encuestas directas en distintos campos pesqueros de la localidad durante 1999. Los ingresos totales por venta en playa se obtuvieron de los avisos de arribo del mismo año.

\section{Análisis de simulación} forma

Se utilizó el modelo de decaimiento exponencial en su

$$
N_{a+1, y+1}=N_{a, y} \cdot \mathrm{e}^{-\left(S_{a} f+S_{a} F_{y}+M\right)}
$$

donde $N_{a, y}$ es el número de organismos de edad $a$ al inicio del año $y, N_{a+1, y+1}$ es el número de organismos de la misma cohorte que sobrevivieron al año siguiente, $f$ es la tasa de mortalidad incidental de juveniles, $F_{y}$ es la tasa de mortalidad por pesca durante el año $y, M$ es la tasa de mortalidad natural, $s_{a}$ y $S_{a}$ son switches que permitieron que $f$ y $F_{y}$ se aplicaran a distintos grupos de edad de acuerdo a los siguientes criterios: $s_{a}=1$ cuando $a<t_{m}$, de otra forma $s_{a}=0 ; S_{a}=0$ cuando $a<t_{c}$, de otra forma $S_{a}=1$. Los reclutas de un año se calcularon con la ecuación $1 \mathrm{y}$ sus parámetros ajustados. Para iniciar el modelo se tomó como entrada la EEI y se asignaron valores semilla para las tasas de 
The benefit-cost $(\mathrm{B} / \mathrm{C})$ ratio was based on the equations:

$$
I_{y}=\sum_{a=t_{c}}^{k} C_{a, y} \cdot W_{a} \pi_{a}
$$

and

$$
O_{y}=E_{y} \cdot \omega
$$

where $I_{y}$ is the total income from first-sales at beach in year $y$; $t_{c}, k, C_{a, y}$ and $w_{a}$ are the same variables as in equation $3 ; \pi_{\mathrm{a}}$ is the price per kilogram at age $a ; O_{y}$ is the total cost of the fishing trips in year $y ; E_{y}$ is the estimated number of trips in year $y$; and $\omega$ is the average cost of a fishing trip. With effort and fishing mortality data from 1999, the catchability coefficient $q$ was calculated considering that $F=q E \rightarrow q=F / E$. The catchability estimated in this way was assumed constant for estimating total costs in other years. The $\mathrm{B} / \mathrm{C}$ ratio was analyzed over time assuming constant prices.

\section{Reference points}

To estimate equilibrium conditions, the model was run keeping $F$ and $f$ constant until population abundance stabilized. This routine was repeated for $f=0$ and $f=0.5 f_{\max }$ combined with $0 \leq F \leq 2$. Three equilibrium points of interest were identified: (1) maximum sustainable yield (MSY), (2) maximum economic yield (MEY), and (3) economic equilibrium yield (EEY).

\section{Simulation scenarios}

Four scenarios were tested by combining two $F$-levels with two $f$-levels. Each level was chosen to represent different management situations. At level $F=F_{\mathrm{EEY}}$, the artisanal fleet is considered unregulated and the fishery tends toward its economic equilibrium level. At level $F=F_{\text {CUR }}$, fishing effort is prevented from increasing, so $F$ is kept as estimated for 2000. At level $f=0.5 f_{\max }$, the shrimp fleet is still by-catching juveniles in the fishing area. At level $f=f_{\text {null }}$, juvenile by-catch is considered completely prevented. For each scenario, 600 simulations for 100 years were run, leaving the model parameters to vary within their 95\% confidence limits assuming a uniform distribution. The model outputs analyzed were: annual yield, $\mathrm{B} / \mathrm{C}$ ratio, stock biomass, and harvest rate. The confidence limits for the outputs were calculated by the equation:

$$
\hat{\theta} \pm \hat{o} z_{a}
$$

where $\hat{\theta}$ is the simulated average of the output, $\hat{\sigma}$ is the standard error of $\hat{\theta}$, and $z_{\alpha}$ is the tabulated $100 \alpha$ percentile (Efron and Tibshirani, 1986). mortalidad $f$ y $F_{y}$, y así se calculó el número de organismos capturados mediante

$$
C_{a, y}=N_{a, y} \frac{F_{y}}{\left(F_{y}+M\right)}\left(1-\mathrm{e}^{\left(-F_{y}-M\right)}\right)
$$

donde $C_{a, y}$ es el número de organismos de edad $a$ capturados en el año $y$. De aquí, el volumen anual de capturas simuladas se estimó con

$$
Y_{S I M, y}=\sum_{a=t_{c}}^{k} C_{a, y} \cdot W_{a}
$$

donde $Y_{\text {SIM a,y }}$ es el peso de la captura simulada para el año $y, w_{a}$ es el peso promedio a la edad $a, t_{c}$ es la edad de primera captura, y $k$ es la edad máxima en la población.

La abundancia de cada cohorte se ajustó año con año variando $F_{y}$ en la ecuación 2 hasta que la captura simulada se igualaba con la captura registrada, es decir $Y_{S I M, y}=Y_{R E C, y}$. Dado que esta condición se cumplió para diferentes valores de $f$, se evaluó la máxima mortalidad de juveniles $\left(f_{\max }\right)$ que la población simulada podía soportar para producir la serie histórica de capturas 1978-2000. De esta forma el modelo de simulación se calibró ajustando los valores de $F_{y} \operatorname{con} f=0.5 f_{\max }$.

La razón beneficio-costo $(\mathrm{B} / \mathrm{C})$ se basó en las relaciones:

$$
I_{y}=\sum_{a=t_{c}}^{k} C_{a, y} \cdot W_{a} \pi_{a}
$$

$\mathrm{y}$

$$
O_{y}=E_{y} \cdot \omega
$$

donde $I_{y}$ es el ingreso total por ventas en el año $y ; t_{c}, k, C_{a, y} \mathrm{y}$ $w_{a}$ son las mismas variables de la ecuación $3 ; \pi_{\mathrm{a}}$ es el precio por kilogramo a la edad $a$; $O_{y}$ es el costo total de los viajes de pesca durante el año $y ; E_{y}$ es el número estimado de viajes en el año $y ; \omega$ es el costo promedio por viaje de pesca. Con los datos de esfuerzo y mortalidad por pesca de 1999 se estimó la capturabilidad $q$, considerando que $F=q E \rightarrow q=F / E$. La capturabilidad estimada de esta forma se consideró constante para la estimación de costos totales en otros años. La razón $\mathrm{B} / \mathrm{C}$ se analizó a través del tiempo considerando precios constantes.

\section{Puntos de referencia}

Para estimar las condiciones de equilibrio se dejó correr el modelo manteniendo $F$ y $f$ constantes hasta que la abundancia de la población se estabilizaba. Esta rutina se repitió para $f=0$ y $f=0.5 f_{\max }$ en combinación con $0 \leq F \leq 2$. Se identificaron 


\section{Results}

\section{Basic parameters}

The length interval observed in commercial catches was 10-99 cm TL, which represents a range of 1.5 to 31 years. Age groups 1 and 2 are not fully recruited to the fishery, and organisms older than 20 years represent less than $0.5 \%$ of the population (fig. 3a). Accumulated length frequency of adults showed that the length at first maturity is $33 \mathrm{~cm}$ TL (fig. 3b), corresponding to 3.4 years. For the simulation analysis, we considered $t_{m}=t_{c}=3$ years and the maximum age was 20 years. No additional groups were considered for simulation analysis.

The age structure showed two different slopes, with the steeper one between 3 and 7 years (fig. 3a). Because $M$ was assumed constant, changes in slope were attributed to $F$,
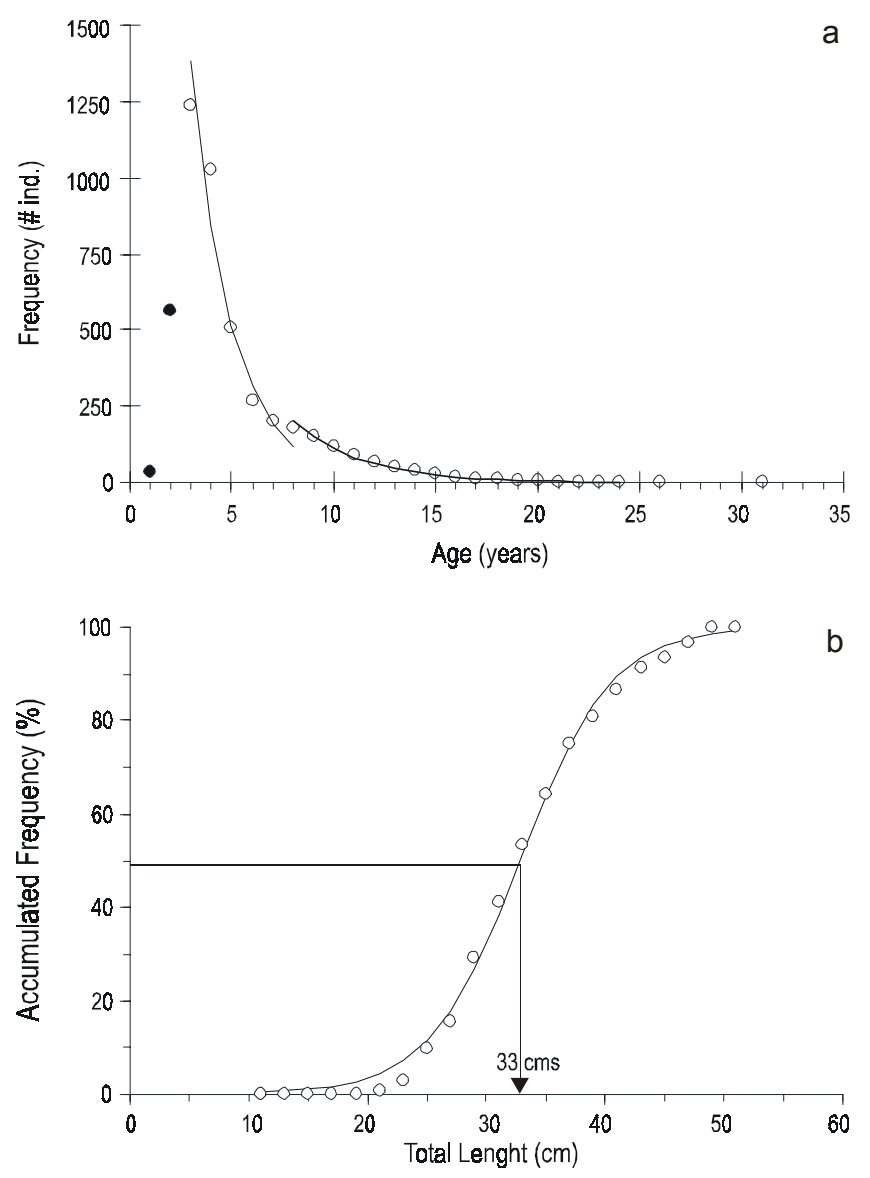

Figure 3. (a) Age frequency of Pacific red snapper in the artisanal fleet catch. Fitted survival curves for age groups 3 to 7 years (thin line) and for 8 years and older (thick line) are shown. Black circles were excluded from fitting. (b) Cumulative frequency of mature individuals by length interval. Estimated length at first maturity is shown.

Figura 3. (a) Frecuencia de edades del huachinango en las capturas de la flota artesanal. Se muestran las líneas ajustadas para los grupos de 3 a 7 años de edad (línea delgada) y para los grupos de 8 años de edad en adelante (línea gruesa). Los círculos negros fueron excluidos del ajuste. (b) Frecuencia acumulada de organismos maduros por intervalo de longitud. Se muestra la longitud de primera madurez estimada. tres puntos de equilibrio de interés: (1) máximo rendimiento sostenible (MSY), (2) máximo rendimiento económico (MEY) y (3) rendimiento de equilibrio económico (EEY).

\section{Escenarios de simulación}

Se probaron cuatro escenarios en los que se combinaron dos niveles de $F$ con otros dos de $f$. Cada nivel se eligió para representar diferentes condiciones de manejo. El nivel $F=F_{\mathrm{EEY}}$ considera la situación en que la flota artesanal se mantiene sin regular y, por lo tanto, la pesquería tiende a su nivel de equilibrio económico. El nivel $F=F_{\text {CUR }}$ considera el caso en que se detiene el incremento del esfuerzo pesquero y $F$ se mantiene igual al estimado en el año 2000 . El nivel $f=0.5 f_{\max }$ considera que la flota camaronera continúa capturando incidentalmente juveniles en la zona. El nivel $f=f_{\text {null }}$ considera la posibilidad de evitar completamente la captura incidental de juveniles. Para cada escenario se corrieron 600 simulaciones durante 100 años, dejando que los parámetros del modelo variaran dentro de sus límites del 95\% de confianza, considerando una distribución uniforme. Los resultados del modelo que se analizaron fueron: el volumen de capturas, la razón $\mathrm{B} / \mathrm{C}$, la biomasa de la población y la tasa de capturas. Los límites de confianza para los resultados se calcularon mediante la ecuación

$$
\hat{\theta} \pm \hat{o} z_{a}
$$

donde $\hat{\theta}$ es el promedio simulado de la salida, $\hat{\sigma}$ es el error estándar de y $z_{\alpha}$ es el percentil $100 \alpha$ tabulado (Efron y Tibshirani, 1986).

\section{Resultados \\ Parámetros básicos}

El intervalo de longitudes observado en las capturas comerciales fue de 10-99 cm LT, lo cual corresponde a un intervalo de 1.5 a 31 años de edad. Los grupos de edad 1 y 2 no están totalmente reclutados a la pesquería y los organismos mayores de 20 años representan menos de $0.5 \%$ de la abundancia de la población (fig. 3a). La frecuencia acumulada de tallas de los adultos mostró que la talla de primera madurez está en $33 \mathrm{~cm}$ LT (fig. 3b), la cual corresponde a 3.4 años de edad. Para el análisis de simulación se consideró que $t_{m}=t_{c}=3$ años, y que la edad máxima es de 20 años. No se consideró ningún grupo adicional para el análisis de simulación.

La estructura de edades mostró dos pendientes diferentes, la mayor de ellas entre los 3 y 7 años de edad (fig. 3a). Dado que $M$ se consideró constante, los cambios en la pendiente se atribuyen a $F$, lo cual significa que los organismos más jóvenes del stock explotable están sujetos a una mayor presión de pesca que los de mayor edad. Dado que la proporción $F_{3-7} / F_{>7}=0.36$, en el modelo de simulación se consideró esta diferencia asignando $S_{a}=0.36$ en la ecuación 2, cuando $a>7$ años. 
meaning that younger individuals from the exploitable stock are subject to greater fishing pressure than the older ones. Since the ratio $F_{3-7} / F_{>7}=0.36$, the difference was considered in the simulation model, assigning $S_{a}=0.36$ in equation 2 when $a>7$ years.

According to the field inquiries, 140 boats in the study area exploit Pacific red snapper. During the fishing season (April to November), each boat makes one fishing trip per day on average; thus we estimated that between 21,000 and 28,000 fishing trips were made in 1999. Field inquiries also showed that the average cost per trip is US\$37 (ranging from US\$16 to US\$74), and that the first-sale price at beach, depending on size, is US\$2.32 per kilogram for small individuals $(0.35-1 \mathrm{~kg})$ and US\$1.27 per kilogram for larger individuals. The rest of the fitted parameters are shown in table 1.

\section{Calibration of the simulation model}

Based on the basic parameters, the historical yield series (1978-2000) could only be simulated within the interval
De acuerdo con las encuestas realizadas, en el área de estudio existen alrededor de 140 embarcaciones que se dedican a la pesca de huachinango. Durante la temporada de pesca (abril a noviembre) cada embarcación realiza en promedio un viaje diario, por lo cual se estima que durante 1999 se realizaron entre 21,000 y 28,000 viajes de pesca. Las encuestas también mostraron que el costo promedio por viaje es de US\$37 (con intervalo de US\$16 y US\$74) y el precio de playa del huachinango depende del tamaño individual: US\$2.32 por $\mathrm{kg}$ los organismos pequeños $(0.35-1 \mathrm{~kg})$ y US\$1.27 por kg los organismos más grandes. El resto de los parámetros ajustados se muestran en la tabla 1.

\section{Calibración del modelo de simulación}

Con base en los parámetros básicos, la serie histórica de capturas (1978-2000) sólo pudo simularse dentro del intervalo $0 \leq f \leq 0.45$. Por lo tanto, el modelo de simulación fue calibrado con $f=0.5 f_{\max }=0.225$ año $^{-1}$ y la mortalidad por

Table 1. Population parameters used to fit the simulation model of Pacific red snapper fishery. The ranges used in the stochastic simulation are given. Tabla 1. Parámetros poblacionales usados para el ajuste del modelo de simulación de la pesquería del huachinango. Se proporcionan los intervalos utilizados en la simulación estocástica.

\begin{tabular}{|c|c|c|c|}
\hline Process & Parameters & Range & Source \\
\hline Age at first catch & $t_{c}=3$ years & Constant & This study \\
\hline Age at first maturity & $t_{m}=3$ years & Constant & This study \\
\hline Growth & $\begin{array}{c}L_{\infty}=97.3 \mathrm{~cm} \\
k=0.111 \text { year }^{-1} \\
t_{\mathrm{o}}=-0.316 \text { year }\end{array}$ & $\begin{array}{c}93.8-100.9 \\
0.103-0.119 \\
(-0.428)-(-0.204) \\
\text { Ranges: C.I. }(\alpha=0.05)\end{array}$ & Rocha-Olivares (1998) \\
\hline $\mathrm{L}-\mathrm{W}$ relationship & $\begin{array}{c}a=1.613 \times 10^{-5} \\
b=2.938\end{array}$ & $\begin{array}{c}1.316 \times 10^{-5}-1.910 \times 10^{-5} \\
2.921-2.955 \\
\text { Ranges: C.I. }(\alpha=0.05)\end{array}$ & $\begin{array}{l}\text { TL }(\mathrm{cm}) \text { vs TW }(\mathrm{kg}) \\
\text { This study }\end{array}$ \\
\hline Mortality & $M=0.201$ & $\begin{array}{c}0.167-0.235 \\
\text { Range: } \max -\min \\
\text { from empirical equations }\end{array}$ & $\begin{aligned} & M_{1}=1.5 k(\text { Jensen, 1997) } \\
M_{2}= & -0.0666+2.52 k(\text { Ralston, } 1987) \\
M_{3}= & e^{-0.0152} \times L_{\infty}{ }^{-0.0279} \times k^{0.6543} \times T^{0.463} \\
& \left(\text { Pauly, 1980) with } \mathrm{T}=17^{\circ} \mathrm{C}\right.\end{aligned}$ \\
\hline Recruitment & $\begin{array}{c}a^{\prime}=1.319 \times 10^{6} \\
b^{\prime}=0.197 \times 10^{6} \\
\sigma^{2}=0.3425\end{array}$ & $\begin{array}{c}\text { Process error } \\
\ln \left(R_{\text {calc }}\right)+w \\
\text { where } w \sim N\left(0, \sigma^{2}\right)\end{array}$ & This study \\
\hline Catchability & $q=1.823 \times 10^{-5}$ & $\begin{array}{l}1.562 \times 10^{-5}-2.083 \times 10^{-5} \\
\text { Range: max-min } \\
\text { from fishing trip variation }\end{array}$ & This study \\
\hline \multirow[t]{2}{*}{ Price } & $\begin{array}{c}\pi=2.32 \mathrm{US} \$ / \mathrm{kg} \text { (small } \\
\text { fish) }\end{array}$ & Constant & $\begin{array}{c}3 \text { to } 5 \text { years }(\sim 0.35 \text { to } 1 \mathrm{~kg}) \\
\text { This study }\end{array}$ \\
\hline & $\begin{array}{c}\pi=1.27 \mathrm{US} \$ / \mathrm{kg} \text { (large } \\
\text { fish) }\end{array}$ & Constant & $\begin{array}{c}\text { Older than } 5 \text { years }(>1 \mathrm{~kg}) \\
\text { This study }\end{array}$ \\
\hline Cost & $\omega=35 \mathrm{US} \$ /$ trip & Constant & This study \\
\hline
\end{tabular}


$0 \leq f \leq 0.45$. Therefore, the simulation model was calibrated with $f=0.5 f_{\max }=0.225 \mathrm{yr}^{-1}$ and the fishing mortality estimated for the year 2000 was used in the simulation scenarios as $F_{\text {CUR }}=0.177 \mathrm{yr}^{-1}$ (fig. 4).

\section{Reference points}

The equilibrium simulations showed that, whenever the juvenile by-catch mortality increases from $f_{\text {null }}$ to $0.5 f_{\max }$, the reference points MEY, MSY and EEY decrease by $45 \%$ and are reached with a lower fishing mortality rate (fig. 4). Therefore, the economic equilibrium scenarios were run with $F_{\mathrm{EEY}}=0.227 \mathrm{yr}^{-1}$ when $f=f_{\text {null }}$ and $F_{\mathrm{EEY}}=0.406 \mathrm{yr}^{-1}$ when $f=0.5 f_{\max }$.

The model fitted to the historical series showed that the yields in 1978-2000 were directly related to fishing mortality. Most of the yield recorded was $\leq 320 \mathrm{t} \mathrm{yr}^{-1}$, just between the equilibrium curves estimated by the model. Yields $>375 \mathrm{t} \mathrm{yr}^{-1}$ were recorded in 1980, 1982, 1996 and 1998, above the equilibrium curves and should be interpreted as extraordinary events (fig. 4).

\section{Simulation analysis}

When $f=0.5 f_{\max }$, the simulation analysis showed that the fishery behaves very similar at two tested $F$-levels. Average simulated yield remained similar to the historical series $(P<0.05)$, while the $\mathrm{B} / \mathrm{C}$ ratio and stock biomass tended to decrease (fig. 5). When $f=f_{\text {null }}$, the fishery behavior differed depending on the $F$-level. If fishing mortality is allowed to reach $F=F_{\mathrm{EEY}}$, the average yield could double in the short term; however, in the long term the $\mathrm{B} / \mathrm{C}$ ratio and biomass are significantly reduced $(P<0.05)$, while the harvest rate

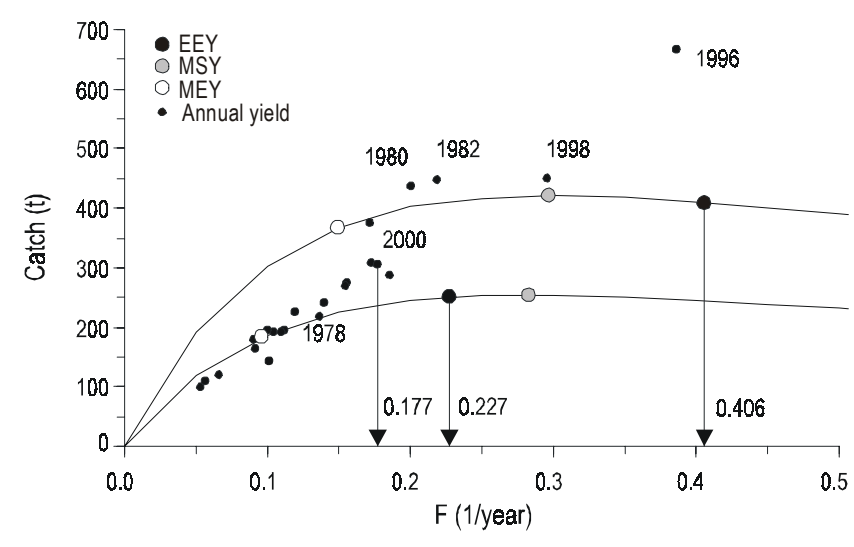

Figure 4. Values of $F$ calibrated with the 1978-2000 historical catch series. As a reference framework, the equilibrium curves at two levels of juvenile by-catch mortality $\left(f_{\text {null }}\right.$ and $0.5 f_{\text {max }}$ ) are shown.

Figura 4. Valores de $F$ calibrados con la serie histórica de capturas 19782000. Como marco de referencia, se muestran las curvas de equilibrio a dos niveles de mortalidad incidental de juveniles ( $f_{\text {null }}$ y $0.5 f_{\text {max }}$ ). pesca estimada para el año 2000 se utilizó en los escenarios de simulación como $F_{\mathrm{CUR}}=0.177 \mathrm{año}^{-1}$ (fig. 4).

\section{Puntos de referencia}

Las simulaciones de equilibrio mostraron que cuando la mortalidad incidental de juveniles se incrementa de $f_{\text {null }}$ a $0.5 f_{\max }$ los puntos de referencia MEY, MSY y EEY se reducen en un $45 \%$ y se alcanzan con una menor tasa de mortalidad por pesca (fig. 4). De esta forma, los escenarios de equilibrio económico se corrieron con $F_{\mathrm{EEY}}=0.227$ año ${ }^{-1}$ cuando $f=f_{\text {null }} \mathrm{y}$ $F_{\mathrm{EEY}}=0.406$ año ${ }^{-1}$ cuando $f=0.5 f_{\text {max }}$.

El modelo ajustado a la serie histórica mostró que las capturas entre 1978 y 2000 estuvieron directamente relacionadas con la mortalidad por pesca. La mayor parte de las capturas fueron $\leq 320 \mathrm{t}$ año $0^{-1} \mathrm{y}$ se ubicaron entre las curvas de equilibrio estimadas por el modelo. Las capturas $>375 \mathrm{t}_{\text {año }}{ }^{-1}$ registradas en 1980, 1982, 1996 y 1998, se ubicaron por encima de las curvas de equilibrio y pueden interpretarse como eventos extraordinarios (fig. 4).

\section{Análisis de simulación}

Cuando $f=0.5 f_{\max }$ el análisis de simulación mostró que en los dos niveles de $F$ probados el comportamiento de la pesquería es muy similar. En promedio, el volumen de capturas simuladas se mantiene similar al de la serie histórica $(P<$ 0.05 ), en tanto que la razón $\mathrm{B} / \mathrm{C}$ y la biomasa del stock tienden a decrecer (fig. 5). Cuando $f=f_{\text {null }}$ el comportamiento de la pesquería difiere entre cada nivel de $F$ probado. Si se deja que la mortalidad por pesca se incremente hasta $F=F_{\mathrm{EEY}}$, el volumen de capturas promedio podría duplicarse en el corto plazo, pero en el largo plazo la razón B/C y la biomasa se reducen significa-tivamente $(P<0.05)$, en tanto que la tasa de capturas se incrementa significativamente a $20-25 \%$ de la biomasa total $(P<0.05)$. Si la mortalidad por pesca se mantiene al nivel de $F=F_{\text {CUR }}$, el estado de la pesquería mejora, ya que el volumen de capturas se incrementa significativamente en un $50 \%$ $(\mathrm{P}<0.05)$. La razón $\mathrm{B} / \mathrm{C}$ y la biomasa del stock tienden también a incrementarse entre un 20-30\% aunque en este caso el incremento no es estadísticamente significativo $(P>0.05)$.

\section{Discusión}

Aunque la importancia de las pesquerías artesanales como fuente de alimento y de empleos ha sido reconocida en todo el mundo, la mayoría de ellas carecen de programas específicos de monitoreo, manejo y regulación (Ralston y Polovina, 1982). Dado que en estas pesquerías rara vez se dispone de información básica para aplicar directamente algún método de evaluación convencional, resulta de vital importancia explorar estrategias alternativas para su análisis. En este sentido, el análisis desarrollado en este estudio representa una alternativa para enfrentar el problema de la escasa información, ya que el 
significantly increases to $20-25 \%$ of the total biomass $(P<0.05)$. If fishing mortality is kept at $F=F_{\mathrm{CUR}}$, the status of the fishery improves because the average yield significantly increases by $50 \%(P<0.05)$. The $\mathrm{B} / \mathrm{C}$ ratio and the stock biomass also tend to increase by $20-30 \%$, although the increment is not statistically significant $(P>0.05)$.

\section{Discussion}

Although artisanal fisheries have been recognized worldwide as an important source of food and employment, most of them lack specific monitoring programs and operate without specific regulations and management programs modelo de simulación integra información biológica disponible (Allen, 1987; Reyna-Trujillo, 1993; Rocha-Olivares y Gómez-Muñoz, 1993; Díaz-Uribe, 1994; Rocha-Olivares, 1998), algunas constantes de los ciclos de vida (Pauly, 1980; Ralston, 1987; Myers et al., 1997, 1999; Jensen, 1997) e información auxiliar sobre la operación de pesca (CES-UABCS, 2000). Esta estrategia nos permitió reconstruir la estructura de edad del stock y evaluar su dinámica poblacional en diferentes escenarios de explotación que difícilmente se hubieran podido probar en la práctica.

Las suposiciones que se utilizaron para la reconstrucción de la estructura de edad son, en su mayoría, las mismas que se utilizan en el Análisis de Población Virtual; es decir, no existen
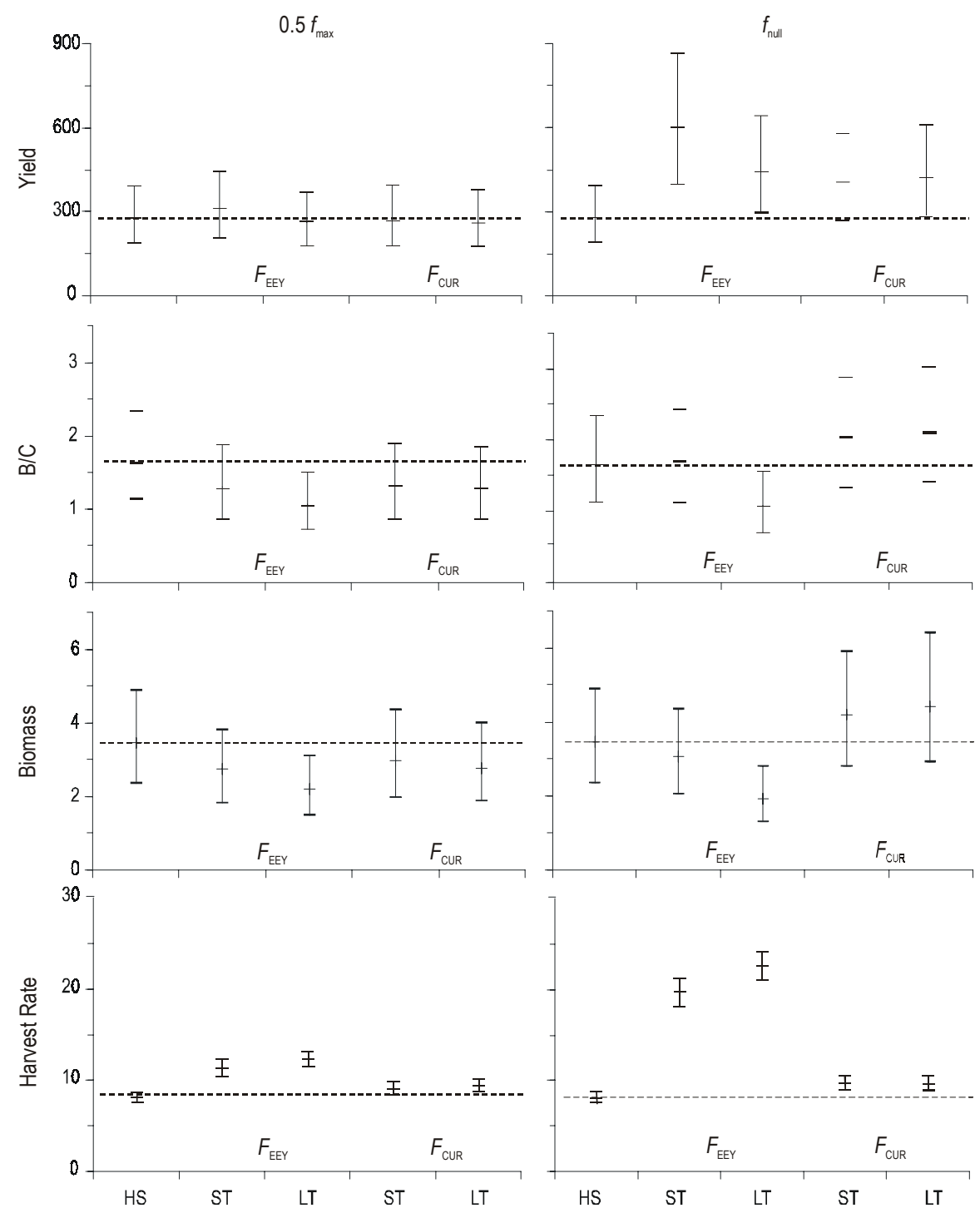

Figure 5. Stochastic simulation outputs: catch volume $(t)$, benefit/cost $(\mathrm{B} / \mathrm{C})$ ratio, biomass $(t \times 1000)$, and harvest rate $(\%)$. The average values and the $95 \%$ confidence intervals of the 600 simulations made at each scenario are presented. Short term (ST) and long term (LT) outputs are shown for each scenario, and they are compared with the historical series average (HS).

Figura 5. Resultados de las simulaciones estocásticas: Volumen de capturas $(t)$, proporción B/C, biomasa ( $t \times 1000)$ y tasa de captura $(\%)$. Se presentan los valores promedio y los intervalos del 95\% de confianza de las 600 simulaciones hechas en cada escenario. Para cada escenario se muestran los resultados del corto plazo (ST) y del largo plazo (LT) y se comparan con el promedio de la serie histórica (HS). 
(Ralston and Polovina, 1982). Because basic information is seldom available for applying conventional assessment methods, it is of paramount importance to explore alternative strategies of analysis in this kind of fisheries. In this sense, the analysis developed in this study represents an alternative to overcome the sparse information problem because the simulation model integrates available biological information (Allen, 1987; Reyna-Trujillo, 1993; Rocha-Olivares and GómezMuñoz, 1993; Díaz-Uribe, 1994; Rocha-Olivares, 1998), lifehistory constants (Pauly, 1980; Ralston, 1987; Myers et al., 1997, 1999; Jensen, 1997), and auxiliary information about the fishing operation (CES-UABCS, 2000). This approach allowed us to rebuild historical age-structure of the stock and to assess its population dynamics under different exploitation scenarios, which cannot be tested in the field.

Most assumptions used for the reconstruction of the age structure are the same as those used in Virtual Population Analysis (VPA), that is, there are no live individuals older than the maximum age, natural mortality rate is known, and there is no net immigration or emigration. Problems and implications derived from these assumptions have been thoroughly discussed in classic literature (Hilborn and Walters, 1992; Sparre and Venema, 1997; Haddon, 2001). An additional assumption in this work is that the fishery started in the year first recorded. This implies that the population abundance in that year was maximum or at least higher than it could be under exploitation pressure. Thus, selection of IAS for calibrating the simulation model was based on the most abundant age structure. Although it is known that the Pacific red snapper fishery started before 1978 , there is evidence of a very low exploitation level in that period. During and before the 1970s, sharks dominated the landings of the artisanal fleets. In that decade, there was also a transition from wind-propelled boats to outboard-motorized boats; hence, before 1978, the deep fishing grounds where Pacific red snapper are caught, were inaccessible to artisanal fleets.

Another assumption in the analysis refers to the initial slope in the recruitment model. For the simulation model, it was established that $\log _{\mathrm{e}} a^{\prime} / b^{\prime}=1.9$, whose value was originally estimated for the northern red snapper Lutjanus campechanus (Myers et al., 1999). This slope represents the maximum recruitment rate $\left(\alpha_{\max }\right)$ when population density is low. This is probably one of the most important population parameters because it is intimately related to the population growth rate $(r)$ and extinction probability, and is frequently used to establish biological reference points for fisheries management (Mace, 1994; Cook et al., 1997; Myers et al., 1997). To estimate this parameter, however, recruitment level should be measured when the stock is at very low abundance, which poses a paradoxical situation (Hilborn and Walters, 1992). Recently, Myers et al. (1999) demonstrated that $\alpha_{\max }$ is relatively constant between species in the same family; thus, without enough information, as in the case of $L$. peru, the value of $\alpha_{\max }$ for a related species can be useful to improve the organismos vivos mayores de la edad máxima, se conoce la tasa de mortalidad natural y no existe inmigración o emigración neta. Los problemas y las implicaciones que se derivan de estas suposiciones han sido ampliamente discutidos en la literatura clásica (Hilborn y Walters, 1992; Sparre y Venema, 1997; Haddon, 2001). Una suposición adicional en este trabajo es que la pesquería se inició el año del primer registro y, en consecuencia, la abundancia de la población en ese primer año es máxima o por lo menos es mayor que cuando se explota el recurso. Por ello la selección de la EEI consideró a la estructura de edades más abundante para la calibración del modelo de simulación. Aunque en el caso del huachinango se sabe que su captura inició antes de 1978, existen evidencias de que el nivel de explotación era muy pequeño en esa época. En la década de los setenta, y años anteriores, los tiburones predominaron en los desembarcos de las flotas artesanales. En esa década también se dio una transición entre la propulsión por viento o remo y el uso de motores fuera de borda, de tal forma que hasta antes de 1978 los caladeros profundos donde se pesca el huachinango eran inaccesibles a la flota artesanal.

Otra suposición en el análisis se refiere a la pendiente inicial del modelo de reclutamiento. Para el modelo de simulación se estableció que $\log _{\mathrm{e}} a^{\prime} / b^{\prime}=1.9$, cuyo valor originalmente fue estimado para el huachinango del Golfo Lutjanus campechanus (Myers et al., 1999). Esta pendiente representa la tasa máxima de reclutamiento $\left(\alpha_{\max }\right)$ cuando la densidad poblacional es baja. Es probablemente uno de los parámetros poblacionales más importantes ya que está íntimamente relacionado con la tasa de crecimiento poblacional $r$ y con la probabilidad de extinción, y se usa frecuentemente para establecer puntos de referencia biológicos en el manejo de recursos pesqueros (Mace, 1994; Cook et al., 1997; Myers et al., 1997). La estimación de este parámetro, sin embargo, requiere de información sobre el nivel de reclutamiento cuando el stock se encuentra en los niveles más bajos de abundancia, lo cual plantea una situación paradójica (Hilborn y Walters, 1992). Recientemente Myers et al. (1999) demostraron que $\alpha_{\max }$ es relativamente constante entre especies de una misma familia, por lo que en aquellas especies donde no se dispone de información suficiente como es el caso de $L$. peru, el valor de $\alpha_{\max }$ de una especie emparentada puede ayudar a mejorar el desempeño del análisis de simulación. Cuando este valor no fue considerado en el ajuste del modelo de reclutamiento, el reclutamiento máximo $\left(a^{\prime}\right)$ estimado fue tres veces mayor y la serie histórica simulada resultó en un incremento de la población durante la fase de explotación. Para un stock que se supone virginal al inicio de la serie histórica, esta situación es contradictoria, por lo que la restricción que se usó con $\alpha_{\max }$ se consideró razonable y consistente.

De acuerdo al modelo calibrado, los volúmenes de captura $>320 \mathrm{t}$ año ${ }^{-1}$ están relacionados a una mayor intensidad de pesca. Dado que $M$ y $q$ se consideraron constantes, estas capturas sólo podrían ser explicadas con un incremento explosivo en el esfuerzo pesquero (fig. 4). Si bien no se cuenta con un 
Table 2. Comparative summary of the exploitation rate $(E=F / Z)$ measured for different Pacific red snapper stocks. Information from the Gulf of Mexico is related to Lutjanus campechanus. Natural mortality $(M)$ is expressed as year ${ }^{-1}$. NA: data not available.

Tabla 2. Resumen comparativo de la tasa de explotación $(E=F / Z)$ medida en diferentes stocks de huachinango. La información del Golfo de México corresponde a Lutjanus campechanus. La mortalidad natural $(M)$ se expresa en año-1. NA: datos no disponibles.

\begin{tabular}{|c|c|c|c|c|c|c|c|}
\hline \multirow[t]{2}{*}{ Region } & \multicolumn{3}{|c|}{ Historical series } & \multirow[t]{2}{*}{ Last year } & \multirow{2}{*}{$\begin{array}{l}\text { Required for } \\
\text { MSY }\end{array}$} & \multirow[t]{2}{*}{$M$} & \multirow[t]{2}{*}{ Source } \\
\hline & Period & Range & Avg. & & & & \\
\hline Gulf of Mexico & Before 1982 & $0.555-0.767$ & 0.662 & NA & $0.444-0.600$ & 0.200 & Ralston (1987) \\
\hline Costa Rica & 1985 & NA & NA & 0.798 & 0.560 & 1.330 & Gutiérrez-Vargas (1990) \\
\hline Guerrero & 1985-1991 & $0.073-0.208$ & 0.149 & 0.208 & 0.480 & 0.380 & $\begin{array}{c}\text { Santamaría and Chávez } \\
\text { (1999) }\end{array}$ \\
\hline Colima & 1980-1988 & $0.476-0.522$ & 0.504 & 0.515 & 0.680 & 0.330 & Cruz-Romero et al. (1996) \\
\hline \multirow[t]{2}{*}{$\mathrm{BCS}$} & 1978-2000 & $0.209-0.659$ & 0.402 & 0.469 & 0.585 (at $\left.0.5 f_{\max }\right)$ & 0.201 & This study \\
\hline & & & & & 0.596 (at $\left.f_{\text {null }}\right)$ & & \\
\hline
\end{tabular}

performance of simulation analysis. When this value was not considered in the fit of the recruitment model, the maximum recruitment $\left(a^{\prime}\right)$ estimated was three-fold higher, and the simulated historical series resulted in growing population abundance during the exploitation phase. For a supposed virgin stock at the beginning of the historical series, this situation is contradictory, so the restriction used for $\alpha_{\max }$ was considered reasonable and consistent.

According to the calibrated model, catch volumes $>320 \mathrm{t} \mathrm{yr}^{-1}$ are related to greater fishing intensity. Since $M$ and $q$ were considered constant, these captures could only be explained by an explosive increment in fishing effort (fig. 4). Although there is no precise record of the fishing effort in the area, national statistics do not show large oscillations in the growth of artisanal fleets (SEMARNAP, 2000b). An alternative explanation is that the highest catches could be related to environmental conditions, since they coincided with the El Niño events of 1982/83 and 1997/98 (SEMARNAP, 2000a). This suggests that the highest catches are possibly the result of higher resource availability. Although the available information on $L$. peru does not allow an explanation about its relation to the environment (Allen, 1987; Gutiérrez-Vargas, 1990; Reyna-Trujillo, 1993; Rocha-Olivares and Gómez-Muñoz, 1993; Díaz-Uribe, 1994; Cruz-Romero et al., 1996; RochaOlivares, 1998; Santamaría and Chávez, 1999; Del MonteLuna et al., 2001), catches $>320 \mathrm{t} \mathrm{yr}^{-1}$ in this part of the Gulf of California should be considered an extraordinary event rather than a potential level of production.

Compared with other stocks, Pacific red snapper from the southwestern Gulf of California has a relatively low exploitation rate (table 2). Nevertheless, we suspect that the fishery tends to reach the economic equilibrium point $\left(F \rightarrow F_{\mathrm{EEY}}\right)$, since the artisanal fleet thus far operates under an open access scheme without specific regulations. On the other hand, although the use of trawling nets inside La Paz Bay was recently banned (Diario Oficial de la Federación, October 30,
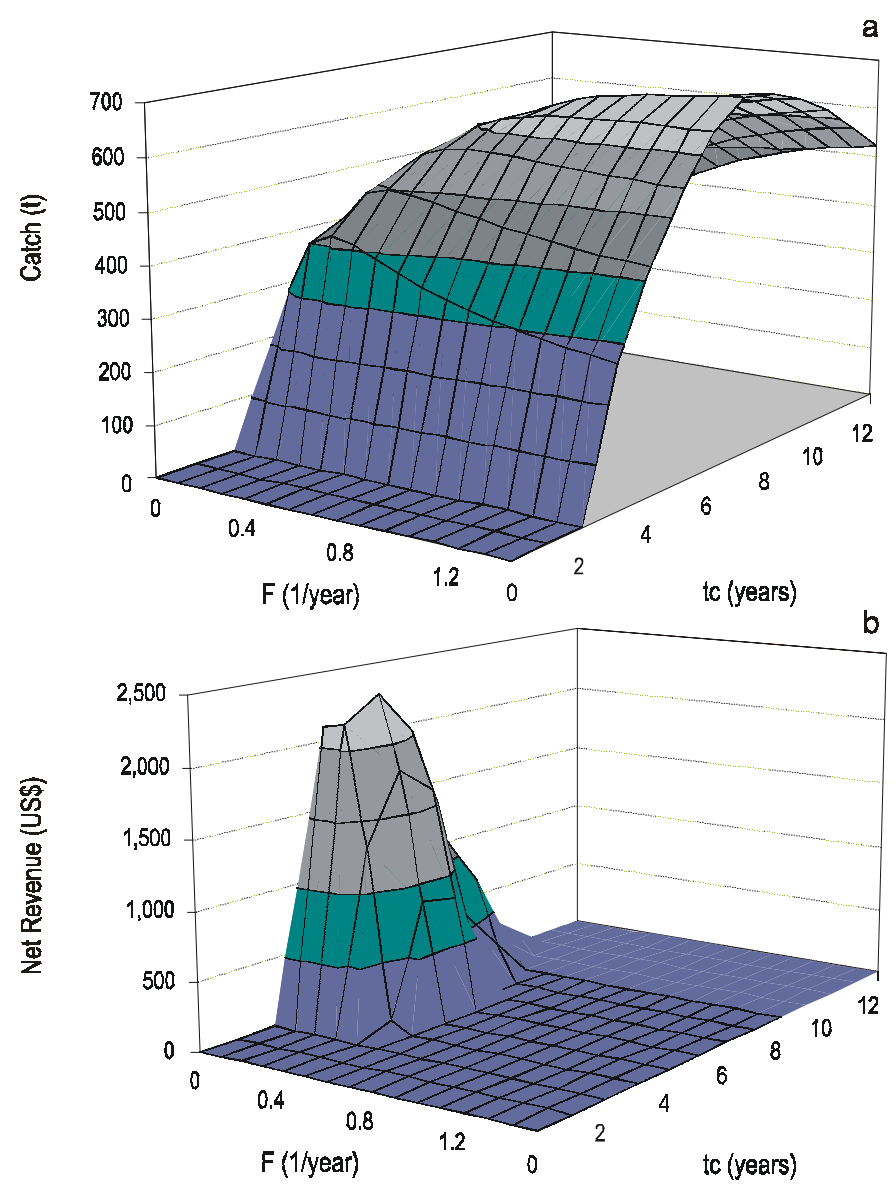

Figure 6. Average long-term outputs for different levels of fishing mortality $(F)$ and age at first capture $\left(t_{c}\right)$ when $f=f_{\text {null }}$ (a) Catch volume $\left(\mathrm{t} \mathrm{yr}^{-1}\right)$. (b) Annual net revenue per boat (US dollars).

Figura 6. Promedios de largo plazo resultados del modelo para diferentes niveles de mortalidad de pesca $(F)$ y de edad de primera captura $\left(t_{c}\right)$ cuando $f=f_{\text {null }}$. (a) Volumen de capturas (t año ${ }^{-1}$ ). (b) Renta neta anual por embarcación (US dólares). 
2002), this measure does not include La Ventana Bay, an important reproductive area for Pacific red snapper (ReynaTrujillo, 1993). Therefore, the by-catch mortality of juveniles has not been totally eliminated from the region. According to the simulation model, under this scenario, the fishery tends to reach MSY or even surpass it. Considering that MSY is no longer regarded as an optimum reference point but a reference limit beyond which the persistence of the population is at risk (Caddy and Mahon, 1996), it is recommended that management measures for the Pacific red snapper fishery in this region be undertaken.

The only possibility for the fishery to develop is to simultaneously regulate the artisanal and shrimp fleets. The problem with this measure is that yield increase would likely not be significantly reflected in the $\mathrm{B} / \mathrm{C}$ ratio or stock abundance (fig. 5). An alternative would be to raise the age of first catch to 4 years (fig. 6). This alternative would imply three concrete management measures: (1) prevent more boats from catching Pacific red snapper in this region, (2) extend restriction on the use of trawling nets to include La Ventana Bay, and (3) modify the selectivity of fishing gear to capture fish larger than $37 \mathrm{~cm}$ instead of $30 \mathrm{~cm}$ TL. These measures could produce several application and control problems, but there are three aspects that, in some way, facilitate their application: (1) restricting the growth of the artisanal fleet does not imply limitations for established fishermen to continue fishing; (2) regional fisheries committees have recently been formed in BCS through which a more direct communication with local fishermen can be established; and (3) perhaps the most important, the status of the fishery seems to be at a level that allows preventive management measures to be undertaken, which in turn, allows a wider margin of negotiation with stakeholders than if restrictive and population recovery measurements were to be taken.

\section{Acknowledgements}

The 1989-1991 samples were obtained from the study "Biología de peces de importancia comercial en la bahía de La Paz y zonas adyacentes", financed by DGICSA-SEP (grants C89-01-0191 and C90-01-0406 to Juan F. Elorduy-Garay). Yield records and fleet operation data came from the study "Diseño de propuestas de co-manejo para pesquerías ribereñas del Golfo de California, en condiciones de sustentabilidad: División BCS", funded by grants from the World Wildlife Fund and Conservation International México to Hernán Ramírez-Aguirre. During the survey, J. Gabriel Díaz-Uribe held a fellowship from the Consejo Nacional de Ciencia y Tecnología for doctoral studies at Centro de Investigaciones Biológicas del Noroeste (CIBNOR). Ernesto A. Chávez and Juan F. Elorduy-Garay received fellowships from the Comisión para el Fomento de Actividades Académicas and Estímulo al Desempeño de Investigación, from the Instituto Politécnico Nacional. We thank Miguel Ángel Cisneros-Mata, Sergio Hernández-Vázquez, Daniel Lluch-Belda, Juana LópezMartínez, and anonymous reviewers for their comments and registro preciso del esfuerzo pesquero en la zona, las estadísticas a nivel nacional no muestran grandes oscilaciones en el crecimiento de las flotas artesanales (SEMARNAP, 2000b). Una explicación alternativa es que las capturas estén relacionadas a las condiciones ambientales ya que coinciden con la ocurrencia de El Niño 1982/83 y 1997/98 (SEMARNAP, 2000a). Esto sugiere la posibilidad de que las capturas mayores son el resultado de una mayor disponibilidad de recurso. Aunque la información que se ha generado sobre $L$. peru hasta el momento no permite explicar su relación con el medio ambiente (Allen, 1987; Gutiérrez-Vargas, 1990; ReynaTrujillo, 1993; Rocha-Olivares y Gómez-Muñoz, 1993; DíazUribe, 1994; Cruz-Romero et al., 1996; Rocha-Olivares, 1998; Santamaría y Chávez, 1999; Del Monte-Luna et al., 2001), las capturas $>320 \mathrm{t}$ año ${ }^{-1}$ en esta región del Golfo de California deben considerarse como un evento extraordinario más que un nivel potencial de producción.

Comparado con otros stocks el huachinango del suroeste del Golfo de California presenta una tasa de explotación relativamente baja (tabla 2); sin embargo, hasta la fecha, la flota artesanal opera bajo un esquema de acceso abierto y sin regulaciones específicas, por lo que se espera que la pesquería tienda a alcanzar el punto de equilibrio económico $\left(F \rightarrow F_{\mathrm{EEY}}\right)$. Por otro lado, aunque el uso de redes de arrastre dentro de la Bahía de La Paz se prohibió recientemente (Diario Oficial de la Federación, 30 de octubre de 2002), la medida no incluye la Bahía La Ventana, que es un área importante de reproducción del huachinango (Reyna-Trujillo, 1993) y, por ende, la mortalidad incidental de juveniles no se ha eliminado por completo en la región. De acuerdo al modelo de simulación, bajo este escenario la pesquería tiende a alcanzar el MSY o incluso rebasarlo. Si consideramos que el MSY ya no se considera un punto de referencia óptimo sino límite, después del cual la persistencia de la población está en riesgo (Caddy y Mahon, 1996), es recomendable tomar medidas de manejo para la pesquería del huachinango en la región.

La única posibilidad de desarrollar la pesquería es mediante la regulación simultánea de las flotas ribereña y camaronera. El problema con esta medida es que muy probablemente el incremento en el volumen de capturas no se vería reflejado significativamente ni en la proporción $\mathrm{B} / \mathrm{C}$ ni en la abundancia del stock (fig. 5). Una alternativa sería incrementar la edad de primera captura a 4 años de edad (fig. 6), lo que implicaría tres medidas de manejo concretas: (1) evitar que se agreguen más embarcaciones para la captura huachinango en la región; (2) extender la restricción del uso de redes de arrastre a la Bahía La Ventana y (3) modificar la selectividad del arte de pesca para que en lugar de capturar organismos a partir de los $30 \mathrm{~cm}$, se capturen de $37 \mathrm{~cm}$ en adelante. Estas medidas conducen a problemas de aplicación y control; sin embargo existen tres consideraciones que de alguna manera facilitan su implementación: (1) la restricción al crecimiento de la flota ribereña no significa restricción alguna para que los pescadores ya establecidos continúen operando; (2) recientemente se instalaron en 
suggestions that helped to improve the manuscript. We also thank the editorial staff at CIBNOR for revising the English manuscript.

\section{References}

Allen, G.R. (1987). Synopsis of the circumtropical fish genus Lutjanus (Lutjanidae). In: J.J. Polovina and S. Ralston (eds.), Tropical Snappers and Groupers: Biology and Fisheries Management. Westview Press, New York, pp. 33-87.

Caddy, J.F. y Mahon, R. (1996). Puntos de referencia para la ordenación pesquera. FAO, Documento Técnico de Pesca, 347 pp.

CES-UABCS. (2000). Diseño de Propuestas de Co-manejo para Pesquerías Ribereñas del Golfo de California en Condiciones de Sustentabilidad. Reporte Técnico. Centro de Estudios Sustentables. Universidad Autónoma de Baja California Sur. La Paz, BCS, México.

Cook, R.M., Sinclair, A. and Stefansson, G. (1997). Potential collapse of North Sea cod stocks. Nature, 358: 521-522.

Cruz-Romero, M., Chávez, E.A., Espino, E. and García, A. (1996). Assessment of a snapper complex (Lutjanus spp.) of the Eastern Tropical Pacific. In: F. Arreguín-Sánchez, J.L. Munro, M.C. Balgos and D. Pauly (eds.), Biology, Fisheries and Culture of Tropical Groupers and Snappers. ICLARM Conf. Proc. 48, pp. 324-330.

Del Monte-Luna, P., Guzmán-Jiménez, G., Moncayo-Estrada, R., Sánchez-González, S. y Ayala-Cortés, A. (2001). Máximo rendimiento sostenible y esfuerzo óptimo de pesca del huachinango (Lutjanus peru) en la Cruz de Huanacaxtle, Nayarit, México. Cienc. Pesq., (14): 159-164.

Díaz-Uribe, J.G. (1994). Análisis trofodinámico del huachinango (Lutjanus peru) en las bahías de La Paz y La Ventana, BCS. México. Tesis de maestría, Centro de Investigación Científica y de Educación Superior de Ensenada, México.

Efron, B. and R. Tibshirani. (1986). Bootstrap methods for standard errors, confidence intervals, and other measures of statistical accuracy. Stat. Sci., 1: 54-75.

Gutiérrez-Vargas, R. (1990). Tasas de crecimiento, mortalidad, reclutamiento, rendimiento y biomasa relativos por recluta de Lutjanus peru (Persiformes: Lutjanidae) en el Pacífico Noroeste de Costa Rica. Rev. Biol.. Trop., 38(2B): 441-447.

Haddon, M. (2001). Modelling and Quantitative Methods in Fisheries. Chapman and Hall, Boca Raton, Florida, 406 pp.

Hilborn, R. and Walters, C.J. (1992). Quantitative Fisheries Stock Assessment: Choice, Dynamics and Uncertainty. Chapman and Hall, New York, 570 pp.

INP (2001). Sustentabilidad y Pesca Responsable en México: Evaluación y Manejo. Instituto Nacional de la Pesca (INP). Secretaría de Agricultura, Ganadería, Desarrollo Rural, Pesca y Alimentación, México, 1111 pp.

Jensen, A.L. (1997). Origin of the relation between K and Linf and synthesis of relations among life history parameters. Can. J. Fish. Aquat. Sci., 54: 987-989.

Lavín, M.F., Beier, E. y Badán, A. (1997). Estructura hidrográfica y circulación del Golfo de California: Escalas estacional e interanual. En: M.F. Lavín (ed.), Contribuciones a la Oceanografía Física en México. Unión Geofísica Mexicana, Monografía 3, pp. 141-171.

Mace, P.M. (1994). Relationships between common biological reference points used as threshold and targets of fisheries management strategies. Can. J. Fish. Aquat. Sci., 51: 110-122.

Myers, R.A., Mertz, J. and Fowlow, S. (1997). Maximum population growth rates and recovery times of Atlantic cod, Gadus morhua.
BCS los comités regionales de pesca, a través de los cuales se puede establecer una comunicación más directa con los pescadores de la localidad y (3), quizá la más importante, que la situación de la pesquería parece estar a un nivel tal que permitiría tomar medidas de manejo preventivas permitiendo un mayor margen de negociación con los usuarios que si fueran éstas restrictivas y de recuperación de la población.

\section{Agradecimientos}

Las muestras de 1989 a 1991 provienen del estudio "Biología de peces de importancia comercial en la bahía de La Paz y zonas adyacentes" financiado por la DGICSA-SEP (subvención C89-01-0191 y C90-01-0406 a Juan F. ElorduyGaray). Los registros de captura y operación de las flotas provienen del estudio "Diseño de propuestas de co-manejo para pesquerías ribereñas del Golfo de California, en condiciones de sustentabilidad: División BCS", financiado por World Wildlife Fund y Conservation International México, bajo la dirección de Hernán Ramírez-Aguirre. Durante la realización del estudio, J. Gabriel Díaz-Uribe recibió una beca otorgada por el Consejo Nacional de Ciencia y Tecnología para estudios de doctorado en el Centro de Investigaciones Biológicas del Noroeste (CIBNOR). Ernesto A. Chávez y Juan F. ElorduyGaray recibieron beca de la Comisión para el Fomento de Actividades Académicas y del programa de Estímulos al Desempeño de Investigación por parte del Instituto Politécnico Nacional. Agradecemos a Miguel Ángel Cisneros-Mata, Sergio Hernández-Vázquez, Daniel Lluch-Belda, Juana LópezMartínez y a los revisores anónimos por sus comentarios y sugerencias que ayudaron a mejorar el contenido del manuscrito. La versión en inglés fue escrita por los autores y revisada por el grupo editor del CIBNOR.

Traducido al español por los autores.

Fish. Bull., 95: 762-772.Myers, R.A., Bowen, K.G. and Barrowman, N.J.. (1999). Maximum reproductive rate of fish at low population sizes. Can. J. Fish. Aquat. Sci., 56: 2404-2419.

Pauly, D. (1980). On the interrelationships between natural mortality, growth parameters and mean environmental temperature in 175 fish stocks. J. Cons. Int. Explor. Mer., 39: 175-192.

Pérez-Mellado, J. y Findley, L.T.. (1985). Evaluación de la ictiofauna acompañante del camarón capturado en las costas de Sonora y norte de Sinaloa, México. En: A. Yáñez-Arancibia (ed.), Recursos Pesqueros Potenciales de México: La Pesca Acompañante del Camarón. Programa Universitario de Alimentos, Instituto de Ciencias del Mar y Limnología, UNAM. Instituto Nacional de la Pesca, pp. 201-254.

Ralston, S. (1987). Mortality rates of snappers and groupers. In: J.J. Polovina and S. Ralston (eds.), Tropical Snappers and Groupers: Biology and Fisheries Management. Westview Press, New York, pp. 375-403.

Ralston, S. and Polovina, J.J.. (1982). A multispecies analysis of the commercial deep-sea handline fishery in Hawaii. Fish. Bull., 80(3): 435-448. 
Reyna-Trujillo, M.M. (1993). Desarrollo gonádico y época de desove del huachinango (Lutjanus peru) Nichols y Murphy, 1922 (Pisces: Lutjanidae) en la Bahía de La Paz, Baja California Sur, México. Tesis de licenciatura, Universidad de Guadalajara, México.

Rocha-Olivares, A. (1991). Edad y crecimiento del huachinango del Pacífico Lutjanus peru (Nichols y Murphy, 1922) (Perciformes: Lutjanidae) en la Bahía de La Paz y zonas adyacentes, BCS, México. Tesis de licenciatura, Universidad Autónoma de Baja California Sur, México, $121 \mathrm{pp}$.

Rocha-Olivares, A. (1998). Age, growth, mortality and population characteristics of the Pacific red snapper, Lutjanus peru, off the southeast coast of Baja California, Mexico. Fish. Bull., 96: $562-574$.

Rocha-Olivares, A. y Gómez-Muñoz, V.M. (1993). Validación del uso de otolitos para determinar la edad del huachinango del Pacífico Lutjanus peru (Perciformes: Lutjanidae), en la Bahía de
La Paz y aguas adyacentes, BCS, México. Cienc. Mar., 19(3): 321-331.

Ross-Terrazas, M.J. (1988). Evaluación poblacional de dos especies de camarón que sostienen la pesquería de alta mar en el norte y centro del Golfo de California. Tesis de licenciatura, Universidad Autónoma de Baja California Sur, México, 70 pp.

Santamaría, A. y Chávez, E.A. (1999). Evaluación de la pesquería de Lutjanus peru (Pisces: Lutjanidae) de Guerrero, México. Rev. Biol. Trop., 47(3): 571-580.

SEMARNAP (2000a). La Gestión Ambiental en México. Secretaría de Medio Ambiente, Recursos Naturales y Pesca, México, 374 pp.

SEMARNAP (2000b). Anuario Estadístico de Pesca 1999. Secretaría de Medio Ambiente, Recursos Naturales y Pesca, México, 256 pp.

Sparre, P. y Venema, S.C. (1997). Introducción a la evaluación de recursos pesqueros tropicales. Parte 1: Manual. FAO Documento Técnico de Pesca, 306/1. 